\title{
Correction to: Guizhi-Shaoyao-Zhimu decoction attenuates rheumatoid arthritis partially by reversing inflammation-immune system imbalance
}

Qiuyan Guo ${ }^{1 \dagger}$, Xia Mao ${ }^{1 \dagger}$, Yanqiong Zhang ${ }^{1 *}$, Shuqin Meng ${ }^{2}$, Yue Xi ${ }^{2}$, Yi Ding $^{2}$, Xiaocun Zhang ${ }^{3}$, Yuntao Dai ${ }^{1}$, Xia Liu ${ }^{3}$, Chao Wang ${ }^{1}$, Yuting $\mathrm{Li}^{1}$ and Na Lin ${ }^{1^{*}}$

\section{Correction to: J Transl Med (2016) 14:165} https://doi.org/10.1186/s12967-016-0921-x Following publication of the original article [1], the authors identified an error in Fig. 7. It was brought to the authors' attention that the cartilage photograph of the MTX group in Fig. 7c was misplaced. The correct Fig. 7 and its accompanying caption are given below. 


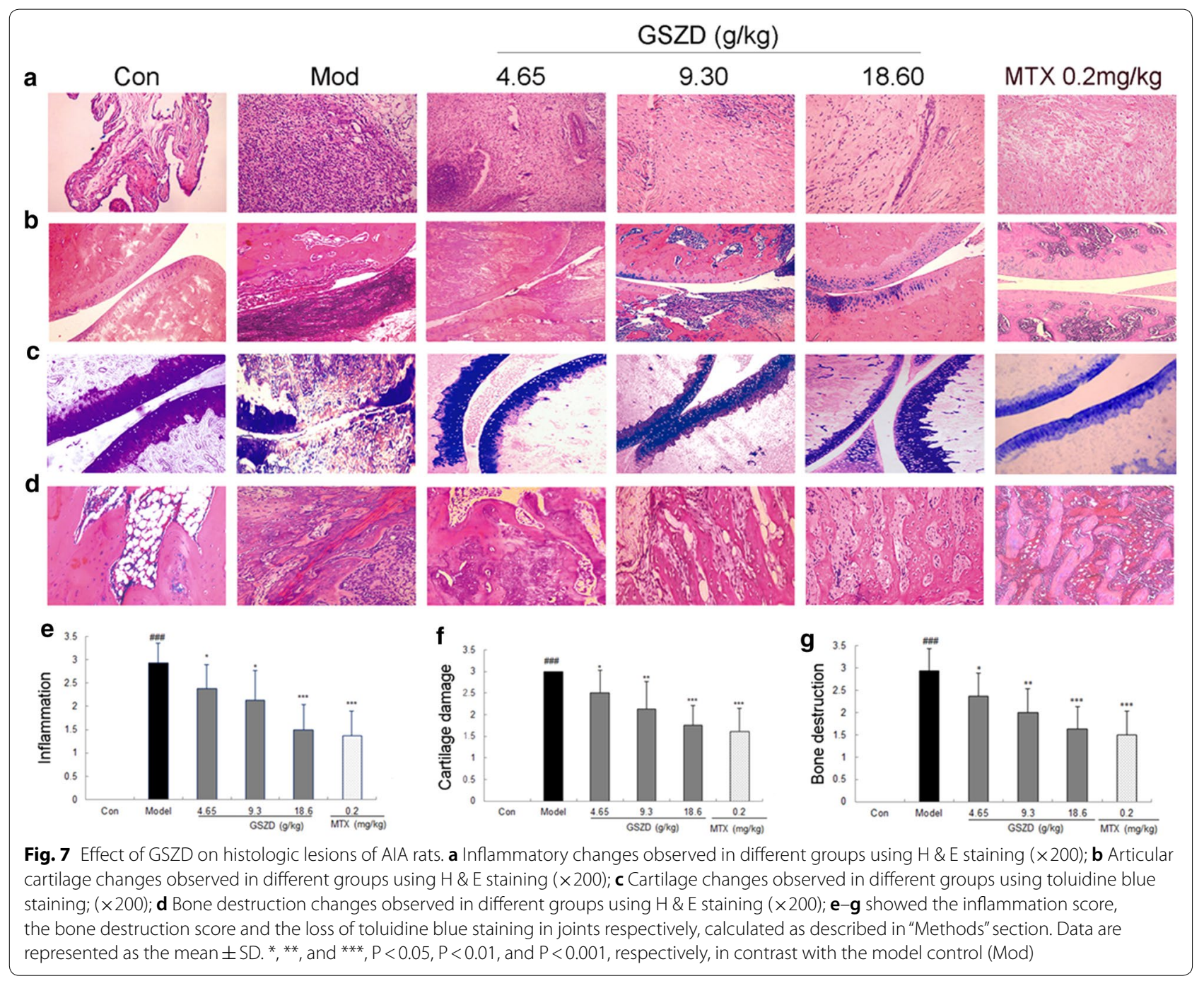

\section{Author details}

${ }^{1}$ Institute of Chinese Materia Medica, China Academy of Chinese Medical Sciences, Beijing 100700, China. ${ }^{2}$ Department of Pathology, Beijing Jishuitan Hospital, Peking University, Beijing 100035, China. ${ }^{3}$ School of Chemistry, Chemical Engineering and Life Science, Wuhan University of Technology, Hubei 430070, China.

Published online: 29 September 2020

\section{Reference}

1. Guo Q, Mao X, Zhang Y, Meng S, Xi Y, Ding Y, Zhang X, Dai Y, Liu X, Wang C, Li Y, Lin N. Guizhi-Shaoyao-Zhimu decoction attenuates rheumatoid arthritis partially by reversing inflammation-immune system imbalance. J Transl Med. 2016;14:165. https://doi.org/10.1186/s12967-016-0921-x.

\section{Publisher's Note}

Springer Nature remains neutral with regard to jurisdictional claims in published maps and institutional affiliations. 